\title{
Protée
}

\section{Le temps décomposé}

Ruines et cinéma

\section{André Habib}

Volume 35, numéro 2, automne 2007

Imaginaire des ruines

URI : https://id.erudit.org/iderudit/017463ar

DOI : https://doi.org/10.7202/017463ar

Aller au sommaire du numéro

\section{Éditeur(s)}

Département des arts et lettres - Université du Québec à Chicoutimi

ISSN

0300-3523 (imprimé)

1708-2307 (numérique)

Découvrir la revue

\section{Citer cet article}

Habib, A. (2007). Le temps décomposé : ruines et cinéma. Protée, 35(2), 15-26. https://doi.org/10.7202/017463ar
Résumé de l'article

Une façon d'aborder « l'imaginaire de la ruine au cinéma " consiste à interroger moins la présence de la ruine au cinéma que la ruine du film en tant que tel et les usages singuliers auxquels elle donne lieu. Cet article se penche sur une " poétique des ruines " contemporaine, à l'oeuvre dans la pratique de plusieurs cinéastes expérimentaux qui explorent les possibilités expressives de la pellicule abîmée ou fragmentée, et sur le singulier " goût de l'archive » qui lui est associé, en insistant tout particulièrement sur un film de Bill Morrison, Light is Calling (2003). L'auteur tente d'analyser la relation entre temps, ruines et cinéma, et de réfléchir aux modalités contemporaines d'une nouvelle mélancolie des ruines. 


\title{
LE TEM PS D ÉCO M PO SÉ RUINES ET CINÉMA
}

\author{
ANDRÉ HABIB \\ [Le cinéma] conserve à contre-temps, parce que le temps \\ cinématographique n'est pas ce qui coule, mais ce qui dure et coexiste.
}

(Deleuze, 1990: 105)

Interroger la relation entre ruines et cinéma, c'est d'entrée de jeu poser la question du temps et de sa perception sensible. Une façon d'aborder «l'imaginaire de la ruine au cinéma» consisterait à s'intéresser moins à la présence de la ruine au cinéma, aux effets de sens et aux types de temporalités qu'elle convoque, qu'à la ruine du film en tant que telle et aux usages singuliers auxquels elle donne lieu. En effet, les fragments du premier cinéma, qui survit souvent en lambeaux et qui porte les marques du passage du temps, donnent lieu depuis une vingtaine d'années à une nouvelle "poétique des ruines", à l'œuvre dans la pratique de plusieurs cinéastes expérimentaux qui explorent les possibilités expressives de ces «vestiges» abîmés ou morcelés. Je voudrais dans cet article me pencher brièvement sur cette tendance du cinéma contemporain et sur le singulier "goût de l'archive» qui lui est associé, en me penchant plus en détail, pour conclure, sur un film de Bill Morrison, Light is Calling (2003) ${ }^{1}$. J'essaierai de proposer l'idée que le cinéma, en raison de l'intimité particulière qui le lie matériellement et ontologiquement au temps, est depuis plusieurs années le site d'une nouvelle mélancolie des ruines, prenant ouvertement - et ce n'est pas un hasard si ces poétiques émergent dans le contexte médiatique actuel - le contre-pied d'une culture numérique qui tend à aplanir toute «différence temporelle» et à effacer les effets de sens, voire le potentiel historique, qui se logent dans la matière-temps du cinéma. Mais avant d'aborder directement ces pratiques, un bref détour historique sur la représentation des ruines au cinéma s'impose. Ce parcours nous permettra de mettre en lumière l'évolution de cette figure et le statut qu'elle occupe à l'intérieur du cinéma moderne d'après-guerre, dans la mesure où, à bien des égards, les pratiques qui nous concernent dans le présent article en prolongent et en radicalisent l'expérience. 

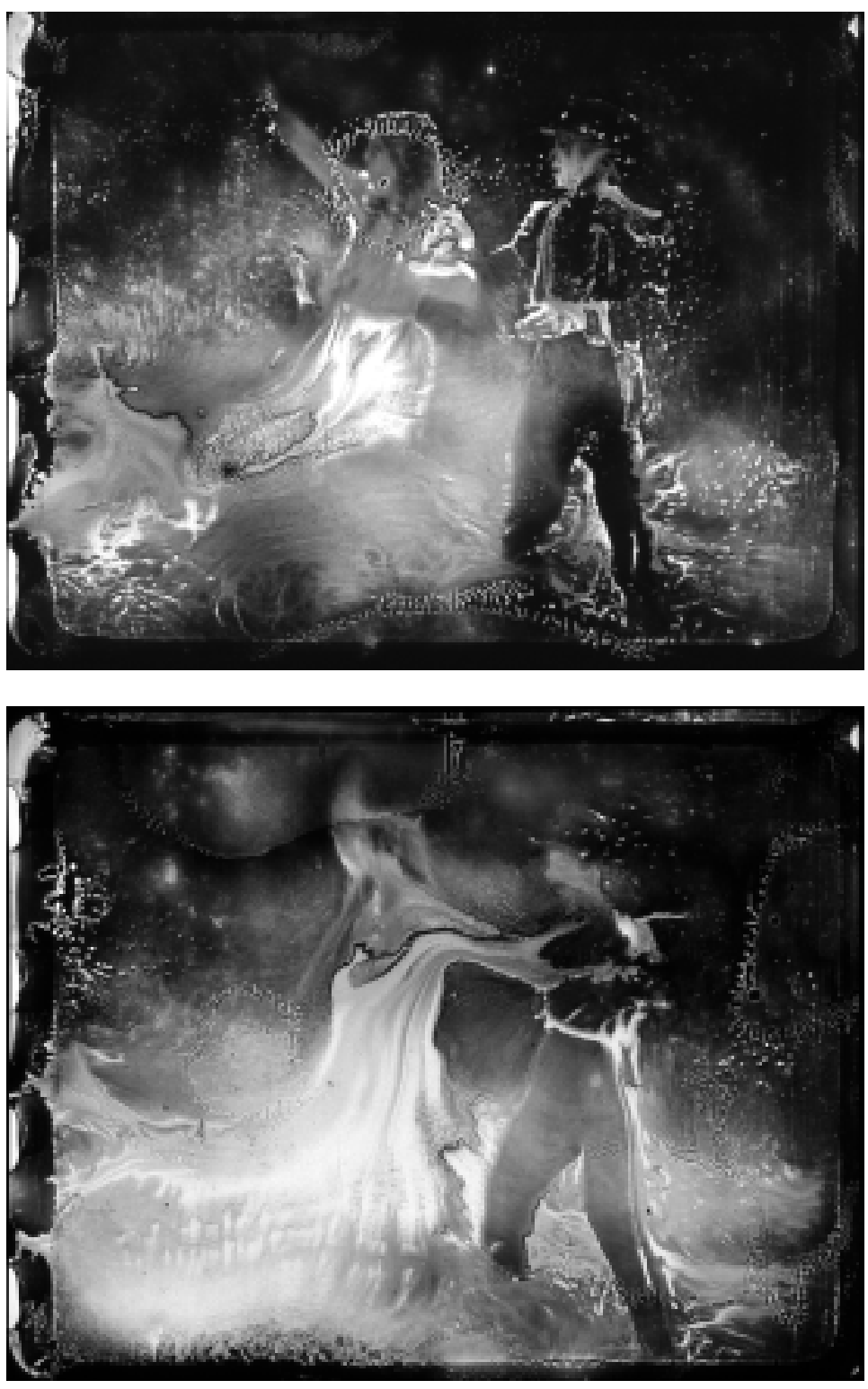

Light is Calling, réal. Bill Morrison, 2003 @ C Hypnotic Pictures 2007. 


\section{DES RUINES AU CINÉMA}

Qu'il s'agisse des vestiges antiques, des décombres de la guerre ou des ruines anticipées, le cinéma a hérité de la peinture, de la littérature et de la photographie et, bien que selon des modalités propres, des différentes figures du temps de la ruine: présence du passé antique (le Pompéi de Rossellini dans Viaggio in Italia [1954], la Roma [1972] de Fellini); désastres contemporains de la guerre (les ruines de Francfort ou de Berlin, chez Wilder, Tourneur ou Rossellini ${ }^{2}$, les ruines de Varsovie recréées pour Le Pianiste [2002] de Polanski); anticipation catastrophique (le Paris en ruines, imaginé par Marker au début de La Jetée [1962], les décombres de New York ou de San Francisco, du Deluge de Felix Feist [1931], à The Day After Tomorrow de Emerich [2003]). Dans chacun de ces cas, qui parfois se chevauchent dans un même film, la ruine est essentiellement ce qui a été filmé dans un décor réel, une reconstitution en studio ou au moyen de la technologie numérique.

Or, force est de constater que, à la différence de la photographie qui, très tôt, s'est trouvé des affinités électives avec la poétique des ruines ${ }^{3}$, le cinéma des premiers temps s'est à première vue peu intéressé aux ruines en elles-mêmes, perçues par les opérateursvoyageurs comme trop statiques pour cet art du mouvement, dominé par le principe de «l'imagemouvement ${ }^{4}$. Dans les premiers films Pathé, Lumière, Edison, on s'attarde plus volontiers aux troupeaux de chameaux en Égypte, aux volées de pigeons sur la place Saint-Marc, à des badauds d'une ville populeuse en Inde, qu'à l'examen de colonnes, de chapiteaux et de temples écroulés. Si les ruines y figurent, c'est comme décor pittoresque d'une action palpitante (Dans les ruines de Carthage, de Victorin Jasset [1907], en est le parfait exemple) ou comme lieu d'une destruction violente (Gli ultimi giorni di Pompei de Mario Caserini [1913]). Car, en retour, la catastrophe (contemporaine ou reconstituée) fascine: nombreuses sont les vues tournées sur le tremblement de terre de San Francisco de $1906^{5}$ ou encore sur l'ouragan qui détruisit Galveston en $1900^{6}$, avec ces longs panoramiques sur la ville en ruine. D'ailleurs, le genre du film-catastrophe (historique ou d'anticipation) apparaît très tôt et offre pour l'essentiel l'image prédominante de la ruine au cinéma, au moins jusqu'à la Seconde Guerre mondiale; après cette dernière apparaîtra un autre mode d'appréhension de la ruine, visible notamment dans les films de Rossellini, Fellini, Pasolini, Wenders, Resnais, jusqu'aux œuvres de Tarr, Sokourov et de Angelopoulos.

S'il faut suivre Gilles Deleuze, la rupture moderne au cinéma, préfigurée dans le néoréalisme italien, consiste à subordonner le temps au mouvement. Une fois «sorti de ses gonds», le temps, disjoint et délié de sa dépendance au mouvement, se donne directement dans l'image comme un temps plié, débordé de l'intérieur par des strates qu'il contient en les redéployant sans cesse. Cette «image-temps», que Deleuze rapporte à l'émergence du cinéma moderne (Welles, Rossellini, Resnais, Visconti), ne serait pas apparue tout de suite pour elle-même, bien que les mouvements aberrants qui révèlent «l'antériorité [du temps] sur tout mouvement normal défini par la motricité» (1985: 54) soient donnés au tout début du cinéma. Or, très tôt, on a cherché à conjurer ces «aberrations» en faisant du temps le «nombre» du mouvement, en le faisant procéder du raccord et de la constitution d'un mouvement ainsi normalisé, en inversant les rapports en quelque sorte. Ce n'est qu'à un détour de son histoire (l'après-guerre) que le schéma sensori-moteur se rompra et qu'apparaitront ces "situations optiques et sonores pures", ces espaces déconnectés, ces états d'errances qui désenchaînent les rapports coordonnés entre la perception et l'action, en nous permettant d'accéder «à cette dimension proustienne d'après laquelle les personnes et les choses occupent dans le temps une place incommensurable à celle qu'ils tiennent dans l'espace» (ibid.: 56). Tout cela était pour ainsi dire en germe dès les débuts du cinéma. Mais il aura fallu «le moderne pour relire tout le cinéma comme déjà fait de mouvements aberrants et de faux-raccords» (ibid.: 59). Cela fait dire à Deleuze que «l'image-temps est le fantôme qui a toujours hanté 
le cinéma, mais [qu']il fallait le cinéma moderne pour donner corps à ce fantôme» (ibid.).

Les visions «fantomatiques» qui s'expriment dans les divers réemplois des images du cinéma des premiers temps dans nombre de films expérimentaux qui m'intéressent (Jacobs, Gehr, Razutis, et plus récemment Morrison, Ricci Lucchi et Gianikian, etc.) sont fortement tributaires de cette influence, de cet éclairage rétroactif du cinéma moderne (sans s'y limiter toutefois). De la même façon, certains films de Garrel, de Rivette, de Ruiz, de Kennett Anger nous invitent à relire les œuvres de Lumière, de Feuillade ou de Méliès, moins - ainsi que l'a longtemps voulu une histoire officielle du cinéma - comme des étapes menant à l'institutionnalisation du cinéma narratif ou documentaire, que comme le tracé d'un mouvement du monde qui procéderait d'une saisie (d'un saisissement) de ce que Schefer appellera «l'êtrefantôme» du temps dans l'image, antérieur à tout mouvement.

En donnant un corps à ce fantôme qu'est «l'imagetemps", on pourrait se demander si le cinéma moderne ne fait pas apparaittre - ou nous rappeler les fantômes qui peuplent, depuis toujours, le premier cinéma et «l'ordinaire» du cinéma en général: c'est-àdire, avant tout, la forme que prend ce trouble, du fait de l'introduction d'un «soupçon" de temps dans l'image. Cette idée, qui nous permet de donner une expansion théorique, historique et médiatique (que Deleuze semble négliger) à la sémiotique déployée dans L'Image-temps, correspond sur bien des points à l'entreprise de Jean-Louis Schefer dans Du monde et du mouvement des images (1997). La pensée de cet auteur, tout à la fois difficile, enchevêtrée, innovante et brillante, s'avère incontournable si nous voulons réfléchir au mode spécifique d'appréhension du temps au cinéma et à sa relation avec la ruine. Si le temps est représenté "directement» dans le cinéma moderne, tel que le théorise Deleuze, la pensée de Schefer nous permet de retraverser, à rebours, le "premier cinéma» et de tirer les conséquences théoriques de cette incursion du temps dans l'image: celle d'un vieillissement du monde, d'une «spectralisation» des corps et des lieux. C'est ce même regard rétrospectif que l'on verra notamment mis en œuvre chez Bill Morrison.

Sans que la lecture de Schefer ne se trouve nommément infléchie par les "fantômes" du cinéma moderne (il n'en parle à aucun moment dans ces termes), il est raisonnable de soutenir qu'elle en porte la trace (du cinéma expérimental d'un Brakhage, par exemple, à certaines œuvres de Garrel ou de Godard). Elle transite par un regard contemporain sur ces images travaillées par le temps et que le temps habite comme sa hantise première, ouvrant ainsi une nouvelle dimension de l'image: celle de sa ruine, de son double spectral. Ce regard rétroactif aurait au moins deux conséquences: il permet d'inscrire la situation contemporaine et une subjectivité historique dans l'appréhension du cinéma (en particulier du cinéma des premiers temps), et, en retour, d'exposer le temps comme un enjeu fondamental de l'invention technique du cinéma aussi bien que des figures et des scénarios qu'il inaugure.

L'étude de Schefer sur le "milieu» de germination du «monde» des images en mouvement recoupe des réflexions sur la littérature (Poe, H.G. Wells), la psychanalyse (Freud), la philosophie (Lucrèce, Vico) et l'histoire de l'art (des peintures rupestres à Matisse). Elle consiste à cerner le creuset d'idées (sur l'univers, le mouvement, les machines mécaniques) dans lequel a pris forme le cinéma - moins comme sa conséquence logique et intentionnelle que dans un partage d'une même constellation, d'un même imaginaire - et ainsi à mieux saisir l'apport propre à son invention. Sa lecture est marquée par l'idée, que l'on retrouve chez Deleuze, d'une antériorité du temps sur le mouvement, puisque, pour Schefer, ce que le cinéma introduit dans l'image, c'est non pas le mouvement, mais le temps. Le mouvement serait même «né comme un accident d'enregistrement du temps, comme son chapelet» (ibid.: 15). Et ce «soupçon de temps» bouleversera radicalement «la durée du monde et sa surface» (ibid.: 10), en prenant le relais de ce qui était déjà en branle - et ébranlé en profondeur - dans l'imaginaire de l'époque. 
L'émiettement de l'univers, que l'on retrouve tant dans les récits fantastiques que dans la décomposition scientifique du monde solide, s'est incorporé, greffé comme une seconde peau - aux «scénarios poétiques» du premier cinéma:

Ils ont intégré au schéma de la vie des émanations (âmes, fumées, souvenirs, fantômes...), c'est-à-dire donné jeu et champ à l'instabilité du monde (et rien n'y a changé: vitesse, rêve, montage haché, superpositions, collages cubistes): les histoires filmées ont intégré les faits de décomposition du monde dans la pensée comme une espèce de loi physique. (Ibid.: 13)

Il pousse au plus loin cette idée dans sa lecture somptueuse de La Chute de la maison Usher (1929) d'Epstein, à partir de laquelle il décline tous les appareils «à enregistrer le temps» convertis en mouvement (ce sont les premiers «appareils de fiction» du cinéma), qui mettent en abyme (et abîment, de l'intérieur) un univers travaillé par cette conscience du temps - matrice scénaristique inépuisable - que le cinéma introduit en en faisant une image. Le film d'Epstein en serait la conséquence logique. Si le cinéma est "une machine à fabriquer du temps», c'est dans la mesure même où il accélère l'œuvre ou le travail du temps sur les choses du monde, en le manipulant, variant durées et mouvement en une dramaturgie inédite: il les décompose en se moulant au mouvement erratique et corrodant de l'univers. Car "[c]ette introduction du temps dans les images est un soupçon ou une possibilité de vieillissement instant du monde" (Schefer, 1997: 76).

La Chute de la maison Usher serait un petit laboratoire présentant, comme à travers une loupe, ce lieu de "pure expérimentation des effets temporels" du cinéma, où le temps est devenu pleinement protagoniste et embraie sur le mouvement destructeur de l'univers (que Schefer rapporte à la révélation de Poe, dans Eureka [1848]). Le film réalise la «mise en ruine poétique du monde», en nous montrant le vieillissement des corps, l'éboulement des lieux, l'atomisation d'un mouvement effréné, l'instabilité et la friabilité de ces rencontres entre les hommes, les choses et les puissances dévorantes de l'univers, des variations de durée, une plasticité du temps. Pour prolonger ces remarques sur Usher, nous dirions que ce ne sont pas seulement les individus et les lieux apparaissant dans un film des frères Lumière, une vue Pathé, un film de Gance ou de l'Herbier, qui sont d'une autre époque, d'une autre histoire: le film luimême, par sa mécanique implacable, nous montre un autre temps, un monde qui, pour cette raison, s'affaisse et se décompose en floculations, qui enveloppe comme d'un voile funeste ce plâtre noirci des corps dansants, qui agite un grain instable, fait trembler une lumière qui rogne les décors et les visages ${ }^{7}$. C'est tout cela qui découle du fait que l'image, dès sa naissance, est, selon Schefer, simplement «donnée au temps, ou elle l'introduit». Schefer poursuit:

[J]amais l'opposition dramatique du temps mangeant ou vieillissant les hommes et les choses à vue d'œil, réduisant l'univers en poussière d'atomes, cela n'avait jamais été une image, tout juste une conscience poétique ou morale.

Enfin, arrangeant des histoires, contes, reportages,

documentaires, le cinéma a montré que l'homme n'était pas contemporain de l'univers, ni même de l'univers des petites choses - il n'a pu, pour raconter quelque chose, qu'accélérer l'un ou l'autre. Ce qui séparait l'homme de l'univers ou faisait radiographie de ce conflit de temps était justement le corps de l'image. (1997: 17)

Et ce corps d'image apparaît en vieillissant ce qu'il enregistre. Le temps ne s'est "pas introduit par un rajeunissement des images", mais en portant immédiatement à l'image «des signes de vieillissement» (ibid.: 15). D'où cette prolifération de fantômes, de revenants, de spectres, d'apparitions, de vampires qui peuplent les fictions des premières années du cinéma - et qui ne cessent de resurgir, depuis, sur les écrans. En même temps qu'il embraie sur les pratiques spirites, les photographies d'auras, les fantasmagories, tout le «continent onirique qui, comme un fuseau dévide sa laine, tisse sa toile depuis le début du XIXe siècle» (ibid.: 116), le cinéma propose une doublure du monde qui se joue et machine «l'être fantôme du temps», comme «sa référence vraie» 
(ibid.: 117). Or, si le cinéma est, de part en part, tout comme la photographie, une "affaire de fantômes", si le cinéma nous présente tant d'«êtres de la survivance» qui s'agitent sur l'écran, si, enfin, on parle volontiers et spontanément de "vieux films", "c'est sans doute, aussi, parce que l'image filmique est, aussitôt, intrinsèquement, menacée de disparition" (Didi-Huberman, 2001: 11).

\section{LES RUINES DE L'IMAGE}

À la fin du XIXe siècle, on a introduit le mot "pellicule» dans le vocabulaire technique de la photographie pour l'opposer à la "plaque» (elle-même très fragile pour la raison qu'elle était en verre). Il est extraordinaire que des hommes aient confié tant d'images, tant d'affects, tant de constructions, tant de beauté, à un support si proche, ontologiquement, de sa propre ruine.

(Ibid. : 13)

À tout ce qui a été dit jusqu'ici sur le temps du cinéma, nous serions tenté d'ajouter, à la suite de Cherchi Usai ${ }^{8}$, que, si la technique cinématographique et photographique traditionnelle dans sa définition la plus stricte consiste à recueillir la trace d'une impression de temps sur de la pellicule, elle est elle-même soumise, avec le temps, à une surimpression du temps, sous forme de décolorations, d'éraflures, de traces de décomposition: la pellicule (photo, cinéma) est "ontologiquement" proche de sa ruine et son espérance de vie est à peine plus longue que l'espace d'une vie (même si cette conscience est «assez nouvelle»). C'est ce que note d'ailleurs Jean-Louis Schefer, au début de son ouvrage:

Ce qui est peut-être aujourd'hui propre à la vie du cinéma n'est pas sa mémoire (son archive) mais la conscience assez nouvelle que cet art extraordinaire a produit des œuvres destructibles et qu'il est une machine à produire et à détruire: c'est un art de "passage» dans nos vies, c'est-à-dire un art de la transition et un dispositif de spectacle à peine moins éphémère qu'une vie humaine. (1997: 6)

Roland Barthes le mentionnait déjà à propos de la photographie qui, «comme un organisme vivant, [...] naît à même les grains d'argent qui germent, [...] s'épanouit un moment, puis vieillit» (1980: 145). C'est sans doute ce constat qui lui fera rajouter - bien qu'il ne fasse pas le lien entre ses deux observations quelques pages plus loin dans La Chambre claire, le Temps comme autre source du Punctum, à côté du «détail». Le détail est de forme, il relève de la composition, alors que le Temps est d'intensité, «c'est l'emphase déchirante du noème ("ça-a-été"), sa représentation pure» (ibid.: 148). C'est cette sensation du Temps qui, pour Barthes, articule l'image sur la mort des sujets représentés, qui les pré-destine à la mort («ils mourront», «ils sont déjà morts»). Il y a donc bien une relation entre la représentation du tempscomme durée et comme fugacité - et la fragilité des supports d'enregistrement dans lesquels le temps est pris, mais continue de devenir, c'est-à-dire de se corrompre, ne fût-ce que virtuellement ${ }^{9}$.

Edouardo Cadava, dans un texte fortement imprégné de Derrida, de Benjamin et de Barthes, écrit: «simultanément construite et effacée, chaque image est une ruine, un lapsus imaginis" (2001: 38; nous traduisons). Sa méditation sinueuse et riche sur la fameuse photographie prise le 23 octobre 1940, trois semaines après le bombardement de la Holland House Library à Londres, repose sur le postulat qu'une image de ruine expose la "vérité» de toute image, la met en abyme en quelque sorte. En retour, une image en ruine, rongée par le temps, dramatise la dialectique de la perte et de la survivance, de la mémoire et de l'oubli, au cœur de chaque image. "Toute image est toujours, en même temps, une image de ruine, une image à propos de la ruine de l'image» (ibid.: 36; nous traduisons).

Le cinéma sans doute complexifie la donne temporelle - déjà complexe - de la photographie. Les «blocs de durée» du cinéma restituent ce que Kracauer appelle le flux de la vie, le «flow of life». On pourrait alors dire qu'en incorporant - en faisant corps avec-le flux temporel de la réalité matérielle, la pellicule cinématographique prend en charge deux temps: une matière-temps prélevée sur du réel (luimême soumis à la pression du temps, à sa finitude), un temps-matière qui constitue sa matérialité 
temporelle. La «momie du changement», selon l'expression consacrée par Bazin, possède, elle aussi, un vecteur de temps; en elle aussi se formule le changement. Le support n'est pas indifférent à ce qu'il supporte, précisément parce qu'il n'est pas quelque chose sur lequel on pose une image. Comme le dit Didi-Huberman, "le support est ce dans quoi l'image a pris. Ce dans quoi l'image est prise et partie prenante» (2001: 11).

Le cinéma matérialise et rend perceptible le temps, non seulement parce qu'il capte, stocke et imprime des durées, qu'il dégage l'entrelacs de temps historiques amalgamés dans chaque image, qu'il périme ce qu'il filme en inscrivant son passage dans des «objets temporels», mais aussi parce qu'il le traduit, dans le corps même du support, sous la forme d'un lent processus de destruction. Le temps rend visibles les marques, inscrites sur la pellicule, de son passage et de sa survivance. La pellicule est elle-même, pour emprunter un mot de Didi-Huberman, une "cendre vivante», une "affaire de fantômes".

À ses débuts, le cinématographe, inutile de le rappeler, était promesse d'immortalité. Plusieurs, suivant le cri du peintre de Poe dans le Portrait ovale (1842), se seraient écriés «c'est la vie elle-même!». Les premiers spectateurs du cinématographe n'auraient pas pu se douter qu'il s'agissait, en vérité, d'une machine à fabriquer de la ruine. Il suffit pour s'en convaincre de relire la presse corporative de l'époque:

Lorsque ces appareils seront livrés au public, écrivait un journaliste en 1895 , lorsque nous pourrons photographier les êtres qui nous sont chers, non plus dans leur forme immobile, mais dans leur mouvement, dans leur action, dans leurs gestes familiers, avec la parole au bout des lèvres, la mort cessera d'être absolue. 10

Ces discours, animés par la nouveauté de la technologie, se sont sans doute périmés assez vite, dès 1907, c'est-à-dire avant même que la pellicule ne manifeste des signes véritables de corrosion, au moment où la dimension «spectaculaire» du cinéma viendra se substituer, du moins dans la conscience populaire, à la mystique spectrale qui anime la recension de Maxime Gorki, revenu du «pays des fantômes». La pellicule d'origine, celle qui avait servi à sa première inscription, possédait à peu près la durée de vie du fils des Lumière faisant ses premiers pas devant le Cinématographe, et bien des films (80\% de la production du cinéma des premiers temps, selon les chiffres récents) auront eu le temps de disparaître avant d'être arrivés à ce terme. Car, par ailleurs, le cinéma est une industrie du spectacle, pour parler comme Malraux.

Naturellement, il est aussi un art de la reproductibilité technique et, si les images des premiers temps du cinématographe survivent et nous parviennent encore aujourd'hui sous une variété de supports, c'est bien à la faveur de contretypages, de restaurations et de transferts numériques qui permettent de débarrasser l'image de certaines de ses scories avant de se retrouver sur nos "petits écrans". Bien que la pellicule d'origine soit lointainement présente, comme "présence d'un lointain», aux supports qui nous sont donnés aujourd'hui pour accueillir les images, et qui tendent bien souvent à annuler leur "épaisseur historique 11 , ces premières images filmées, au début du siècle, parviennent à conserver cette spectralité, ce que j'appellerai leur caractère, auratique, de revenance ou de survivance. $\mathrm{La}$ revenance serait plus précisément ce qui résiste là où le temps a fait son œuvre, ce qui montre les signes à la fois d'une déréliction et d'un devenir. C'est ce temps paradoxal - qui n'est autre que le temps de la ruinequi nous place au plus près de la spécificité du temps du cinéma (même si cette idée, aujourd'hui, semble elle-même vouée à disparaître et n'aura été que d'un temps). Si le cinéma archive du temps et du mouvement, il doit souvent, par le fait même, comme l'avait vu Epstein, «paye[r] la rançon de son privilège exclusif, d'exprimer la mobilité» ${ }^{2}$.

\section{ARCHIVE ET DISPARITION}

Les archivistes, les restaurateurs et les historiens du cinéma (tout particulièrement des premiers temps) ont appris à composer avec le fragmenté, le décomposé, bien que, pour plusieurs d'entre eux, le 
travail consiste à combler ces manques au mieux des connaissances, à effacer ou faire fi des traces délitantes qui se sont déposées sur le matériau filmique, à colmater l'incomplétude des éléments par des sources textuelles ou autres, quitte à condamner à l'oubli des pans entiers de collections où les bobines sont tantôt non identifiables, tantôt trop endommagées.

Il existe toutefois une autre utilisation du fragment et des ruines filmiques, appelée de leurs vœux par quelques rares archivistes et historiens du cinéma ${ }^{13}$, mais que l'on trouve surtout chez des artistes et des cinéastes dits expérimentaux, chiffonniers-plasticiens dont les œuvres sont apparues depuis les années 1980. Ces œuvres interrogent de diverses façons l'empreinte du temps sur la matière-mémoire de la pellicule, participant ou réagissant, c'est selon, à un Zeitgeist du cinéma contemporain, à l'époque de sa paradoxale mise au patrimoine et à l'aube de sa soi-disant disparition, alors que nous nous enfonçons dans ce que l'historien et archiviste Paolo Cherchi Usai a appelé il y a quelques années le «Digital Dark Age».

Je pense en particulier à l'éventail de films faits de pellicules trouvées, found footage selon l'expression consacrée, qui usent, recyclent, détournent parfois des fragments des premiers temps du cinéma pour en faire des œuvres «originales». Ces artistes mêlent au "goût de l'archive» une esthétique, souvent mélancolique, de la ruine et de la disparition.

Apparus depuis une vingtaine d'années tout au plus, ces films explorent les voies d'une véritable "archéologie poétique», à mi-chemin entre le film de compilation et le cinéma expérimental: c'est du moins ainsi que l'on peut sommairement caractériser les œuvres de Angela Ricci Lucchi et Yervant Gianikian, certains films de Peter Delpeut, Gustav Deutsch ou Bill Morrison. Ces œuvres partagent un certain nombre de traits communs: en général, les images originales sont retirées de leur contexte narratif spécifique et viennent faire affleurer un temps englouti. Elles s'intéressent plus précisément aux effets saisissants de la décomposition ou du vieillissement de la pellicule nitrate (interdite dans les années 1950), aux effets de sens qu'engendre la juxtaposition de fragments hétérogènes, ou encore à l'inconscient visuel, aux symptômes d'une époque que le ralentissement, le recadrage, la décomposition du mouvement révèlent.

Ces artistes ont ceci en commun qu'ils trouvent leur matériau de base - et c'est souvent leur unique matériau - dans des archives (institutionnelles, publiques, corporatives), ou encore dans des stocks rachetés de collectionneurs. Ces images, fréquemment rongées par la moisissure et l'usure, sont, selon le cas, transférées sur un support pellicule ou numérique, elles sont remontées, rephotographiées, travaillées à la tireuse optique et parfois (dans le cas de Ricci Lucchi et Gianikian notamment) recadrées, teintées, solarisées ou coloriées ${ }^{14}$.

Dominique Paini, dans l'une des classifications chronologiques hâtives mais utiles dont il a le don, avançait l'idée suivante: au moment où se constituèrent les premières cinémathèques, au début des années 1930, il s'agissait avant tout de sauver les films pour leur valeur artistique. Dans les années 1960, ce fut pour leur valeur documentaire, leur puissance de témoignage. Enfin, depuis les années 1980 (l'année 1980, rappelons-le, fut votée "année du patrimoine»), ce fut pour leur valeur matérielle: tout fragment de pellicule est désormais marqué du sceau d'une mémoire qu'il faut sauver à tout prix (Païni, 2002: 25 26). D'où, il me semble, les diverses élégies ou «célébrations du nitrate» (Smither, 2002), un certain engouement esthétique pour les films amateurs et de famille, qui privilégie bien souvent moins le contenu des images, leur indicialité, que les propriétés plastiques du support qui viennent signifier le passé.

Si tout film est matière et mémoire, il semble que, dans ces films, la matière soit devenue mémoire. Ces images font corps avec leur support, engendrant une indiscernabilité - qui produit ses propres effets de sens - entre ce qui relève de l'enregistrement et ce qui relève du matériau. Cela est particulièrement évident dans ces cas où les films semblent se décomposer et apparaissent, comme l'exprime Peter Delpeut au début de Lyrical Nitrate, "en train de disparaître sous nos yeux». En rendant visible les forces matérielles du 
temps, on provoque un véritable, singulier et surprenant devenir-image de la matière écroulée.

Pour reprendre une idée que Jean Starobinski avait formulée à propos de la mélancolie des ruines au XVIII siècle, ces œuvres ont "partiellement survécu à la destruction, tout en demeurant immergé[es] dans l'absence" (1964: 180). Leur mélancolie réside peutêtre «dans le fait qu'elle[s] [sont] devenue[s] [des] monument $[\mathrm{s}]$ de la signification perdue» (ibid.). Ces fragments de films repris apparaissent en tant qu'image et trace, puisqu'ils ont été dépossédés de leur fonction et de leur signification initiale, documentaire ou fictionnelle (beaucoup des éléments sont anonymes, plus ou moins datés). Ils ne servent plus à rien, mais apparaissent d'autant plus dans leur unicité «auratique».

\section{Light is CALling De BiLl Morrison}

Light is Calling de Bill Morrison représente un bon exemple des paradoxes et de la fécondité de cet imaginaire de la ruine filmique. En 2001, des employés de la Library of Congress à Washington présentent au cinéaste new-yorkais Bill Morrison - qui venait de finir Decasia. A Symphony of Decay (2000) une copie nitrate fortement abîmée d'un film du cinéaste James Young, The Bells (1926), avec Boris Karlof et Lionel Barrymore, d'après une nouvelle de Poe, qu'ils étaient sur le point de détruire (ils possédaient un négatif d'origine qui, lui, était bien conservé et en parfait état).

En réimprimant à l'aide d'une tireuse optique chaque photogramme afin de ralentir le défilement des images et de mettre en valeur les effets plastiques engendrés par la décomposition de l'émulsion, Morrison remonta, à partir de cette copie nitrate d'origine, deux films originaux: The Mesmerist et Light is Calling, respectivement en 2002 et 2003. Une musique de Bill Frisell accompagne le premier; une pièce originale de Michael Gordon (compositeur de Decasia), le second.

The Mesmerist conserve une certaine trame narrative (tout en transformant considérablement celle du film de Young): un aubergiste se fait

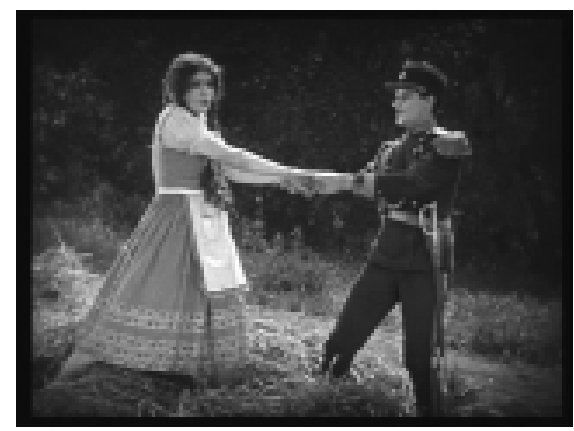

The Bells, réal. James Young, 1926 @ Image Entertainment, 2007.

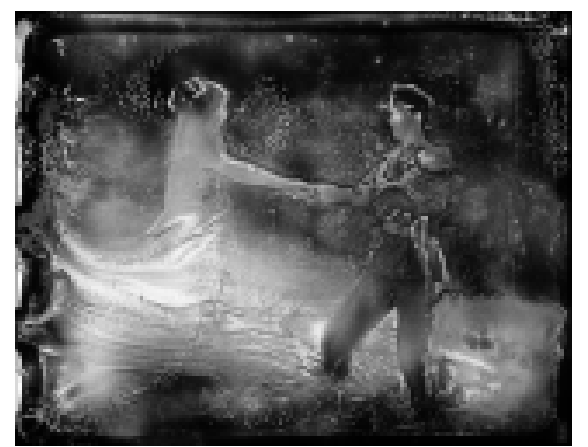

Light is Calling, réal. Bill Morrison, 2003 @ ) Hypnotic Pictures 2007.

hypnotiser lors d'une fête foraine; en flash-back (qui n'est pas dans le premier film), on découvre que cet homme a en fait assassiné et volé un riche voyageur juif, des années auparavant. Le passé revient le hanter à mesure que la décomposition de la pellicule envahit l'image et que le film se transforme lui-même en vision cauchemardesque.

Tout différemment, Light is Calling est extrait d'une séquence unique, beaucoup plus courte et marginale par rapport à l'intrigue principale, et sa trame narrative est infiniment moins développée. Dans l'une des scènes du film de Young, un officier de cavalerie courtise la fille de l'aubergiste, tombée d'une charrette de paille dans laquelle elle se cachait. Il parvient à rapidement la séduire et la convainc de filer avec le régiment. Cette scène, qui, au complet, dure à peine deux minutes dans le film original, est métamorphosée dans Light is Calling, par un procédé complexe, en un riche poème visuel de huit minutes accompagné par une partition pour orchestre de Michael Gordon. 
Pour réaliser ce film, chaque photogramme du film original a été multiplié quatre fois à la tireuse optique. Chaque série de quatre photogrammes a ensuite été surimposée avec les deux premiers photogrammes de la série suivante, et ainsi de suite. Cela produit un étrange effet de fondu enchaîné continu et contribue à cette impression de flottement fantomatique sur laquelle repose l'efficace visuelle du film. Light is Calling est ainsi, en quelque sorte, le double redoublé et le décalque spectral du film de Young: une histoire de fantômes, ou une histoire, au temps des fantômes, devant laquelle on serait tenté de reprendre cette citation de Nicole Brenez à propos des films de Ricci Lucchi et Gianikian:

D'un photogramme à l'autre, les apparences contestent, minent, ruinent l'essence; cette silhouette que spontanément on ramène à l'identification d'un corps n'est qu'une ébauche dont chaque miroitement photogrammatique renvoie à la disparition, à l'effacement et à la mort. (1998: 321)

Le découpage de l'action, comme dans le film de Young, se résume à quelques traits minimaux: plans de la cavalerie, jeux de regards de la jeune fille et du jeune homme, rencontre du couple au centre de l'image, sortie du cadre par la droite. Cette description n'épuise pas toutefois ce qui se passe dans le film de Morrison, et qui a précisément à voir avec ce qui est arrivé à (et sur) la pellicule. Avec la décomposition du nitrate sont apparus de larges trous, crevasses, bouillons roses, blancs, pourpres, noirs, qui ont modifié le mouvement des corps et l'expression des visages, dégoulinant ou éclatant en girations spectaculaires, pris dans la «vague nébuleuse» (ou la "nébuleuse vague») de l'émulsion décomposée.

Tout comme, à une autre époque, ces pierres imagées d'agate ou de marbre dont parlent Jurgis Baltrusaitis et Michel Makarius ${ }^{15}$, la pellicule ruinée crée des formes "naturelles», «engendrées par le temps", qui se prêtent au jeu des analogies et des métaphores: nuages, bourrasques, vagues gluantes, créatures ectoplasmiques, surdéterminant ou détournant parfois le sens des scènes représentées. Les effets d'empâtement, de lavis, de coulures que le hasard a produits rompent la continuité des gestes et développent, en autant de tableaux réenchaînés, une nouvelle «intrigue», plus énigmatique, par-dessus la première, tantôt féerique ou onirique, tantôt cauchemardesque ou horrifiante, évoquant tour à tour les têtes torturées des polyptyques de Francis Bacon, une valse de fantômes ou l'iconographie des contes de fées.

La technique de réimpression que Morrison a employée dilate et complexifie la donne du temps. En effet, chaque photogramme se dédouble, pour passer à l'image suivante, en présent actuel et déjà-passé, surimprimé: chaque image génère de l'intérieur en quelque sorte sa propre succession ou, plutôt, chaque image actuelle se trouve nimbée de sa propre image virtuelle qu'elle fait jaillir. Pour parler comme Deleuze, chaque "perception actuelle s'entoure d'une nébulosité d'images virtuelles qui se distribuent sur des circuits mouvants de plus en plus larges, qui se font et se défont» (1996: 179-180). La "mélancolie des ruines» dans ce film - que l'élégiaque violon de Gideon Kramer accentue - provient en bonne partie de ce rythme particulier, de cet intervalle de temps, suspendu et s'évanouissant, s'élevant et s'abolissant dans ses propres effets de mutation, dans un double mouvement de stase et de reprise.

Cette coalescence des temps de l'image crée un effet visuel tout particulier, puisque la succession plus ou moins régulière des images filmées (la scène à proprement parler) se trouve sans cesse agitée par la scansion irrégulière des motifs générés par la décomposition de la pellicule. La décomposition vient accentuer le «fait photogrammatique» du film: elle représente le mouvement décomposé, tout en produisant une recomposition du mouvement: celui du cavalier et de la jeune fille se déplaçant dans le cadre, mais aussi celui de l'animation des couleurs, les ondulations, les effets d'embrasement sur la pellicule. Ainsi, ce film convoque et met en jeu un certain nombre de temps: ces images apparaissent comme ce qui a effectivement duré malgré l'usure, qui a été sauvé; en même temps, ce défilement d'images coexistantes a 

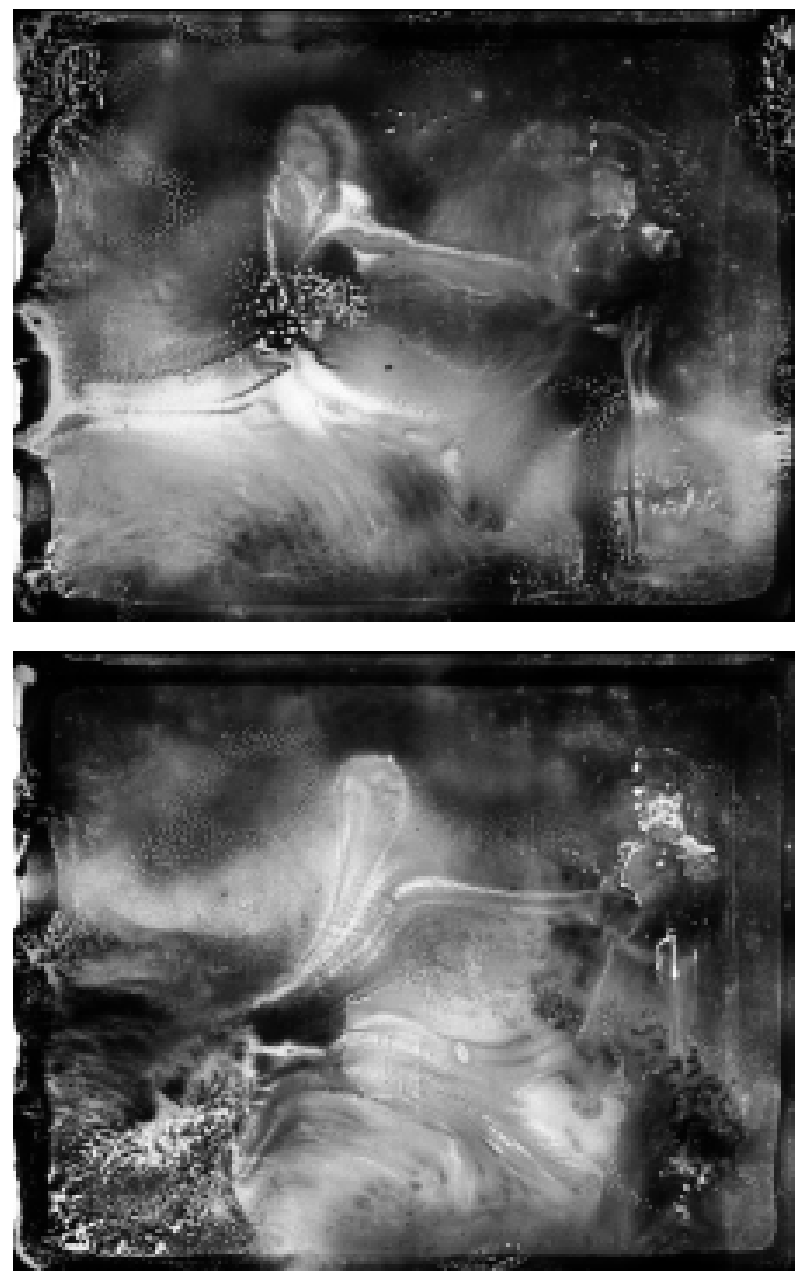

Light is Calling, réal. Bill Morrison, 2003 @ Hypnotic Pictures 2007.

quelque chose de fugace, d'insaisissable, ces dernières disparaissant aussitôt apparues.

\section{CONCLUSION}

Ces œuvres en ruine nous en disent, au bout du compte, moins long sur les films qu'elles réemploient, que sur notre propre relation au temps, aux images et à leur histoire actuelle. Elles participent d'une vaste constellation de pratiques et de questions (patrimoniales, technologiques, institutionnelles), dont il était évidemment impossible de donner ici tous les contours, mais qui forme un pan incontournable de cet imaginaire contemporain de la ruine au cinéma.

Ces films, pour finir, nous invitent à envisager le «temps vécu » du sujet-spectateur de cinéma autrement que dans les termes de la narratologie, qui conçoit toujours le temps en fonction d'un «temps pour comprendre" trouvant son fondement dans le jeu ou le pacte fictionnel ou documentaire. Il faudrait penser plutôt un mouvement dans le temps, entre les strates de temps des images. C'est cette relation au temps qu'une archive du film - ou, plutôt, que le cinéma comme archive - conserve et que ces films en ruine réexposent. Ces palimpsestes des temps exposés, présents et passés, instaurent une nouvelle mélancolie spectatorielle, une mélancolie des ruines, propre au cinéma, née du temps, de son archive et de son anachronique actualité.

\section{N O TES}

1. Des portions de cet article ont été développées à partir de la présentation du dossier visuel que j'avais consacré à Light is Calling de B. Morrison, paru dans la revue Intermédialités en 2004.

2. Berlin Express (J. Tourneur, 1948), A Foreign Affair (B. Wilder, 1948), Germania anno zero (R. Rossellini, 1947).

3. On lira entre autres sur ce point $\mathrm{M}$. Caraion, particulièrement la deuxième partie de son ouvrage, "Un objet photogénique entre science et rêverie: la ruine", consacrée aux photographies de Maxime Du Camp et d'Auguste Salzmann en Orient (2003 : 269-317).

4. C'est notamment la conclusion de M. Bertozzi dans son étude sur l'image de l'Italie et de Rome au cinéma (2001).

5. Le tremblement de terre de San Francisco donna lieu à quelques reconstitutions, dont cette surprenante maquette de la ville en feu: San Francisco Disaster (Mutoscope et Biograph, 1906), que l'on peut visionner sur le site de la Library of Congress. En ligne: http:// 
memory.loc.gov/mbrs/lcmp003/m3b01123.mpg (page consultée le 3 mai 2007).

6. Voici une description du catalogue de distribution Edison pour le Cycle de films tournés à Galveston: "Ce terrible désastre qui a choqué le monde entier a laissé une impression indélébile dans la conscience du public. Tous seront intéressés à voir des vues animées authentiques de cette ville américaine type, presque complètement effacée par les forces combinées du vent et de l'eau. Les films compris dans notre liste sont authentiques, et constituent les seules vues animées enregistrées pendant que la ville de Galvestone baignait dans le chaos " (Edison film catalogs, $\mathrm{n}^{\circ} 105$, juillet 1901: 14-15; nous traduisons).

7. Ces remarques peuvent faire écho à cette très belle interrogation de Bazin, à propos du film de compilation d'images de la Belle Époque de Nicole Vedrès, Paris 1900 (1947): «Pourquoi les transpositions de valeur involontaires exclusivement dues à l'émulsion de la pellicule utilisée à l'époque, rendent-elles le coin de jardin où est en train de peindre Monet exactement semblable aux plus impressionnistes des tableaux du peintre?» (Bazin, 1998: 243).

8. "Cinema is the art of destroying moving images", est l'axiome principal de P. Cherchi Usai dans son ouvrage The Death of Cinema (2001: 7).

9. Il est frappant de constater que Barthes ne fait, à aucun moment, le lien entre la fragilité du support, son caractère organique et la mort des individus qu'anticipe la photographie.

10. La Poste, 30 décembre 1895, cité dans E. Toulet (1988: 135).

11. «La perfection de la réparation ou de la reconstitution conduit à abolir ce qu'il est convenu de nommer l'épaisseur historique, c'est-àdire les traces sensibles de l'écoulement du temps sur la pellicule. [...] [C]es techniques donnent le sentiment de pouvoir effacer l'intervalle entre la création de l'œuvre et la réactualisation restauratrice, intervalle qui peut être interprété comme une période vide (Païni, 2002: 92).

12. Cité dans A. Méniel (1991: 66).

13. Voir sur ce point E. de Kuyper (1992: 45). L'initiative menée par le Filmmuseum d'Amsterdam de monter et de présenter des fragments de films anonymes, souvent très courts, regroupés sous le titre Bits and Pieces, s'inscrit dans le même esprit.

14. Sur les procédés employés par les deux cinéastes, voir $\mathrm{S}$. Toffetti (1992).

15. Sur ce point, voir J. Baltrusaitis (1983: 54-88). Au XVIe siècle, on voit apparaître une fascination pour la "décrépitude", «les formes instables, provisoires, changeantes ", bref pour le mouvement de la vie et de la mort, de l'artificiel et du naturel, en particulier entre le géologique et le végétal. D'où la fascination pour les "pierres de ruines" ou «pierres de Florence» (nommées pietra cittadina), en raison de la ressemblance qu'elles arborent avec un paysage de ville en ruine, que certains artistes utiliseront comme surface pour y peindre des figures. Sur ce point, voir M. Makarius (2004: 50-51).

\section{RÉFÉREN CES BIBLIO G RAPHIQ UES}

BALTRUSAITIS, J. [(1957) 1983]: Aberrations: essai sur la légende des formes. 1. Les Perspectives dépravées, Paris, Flammarion, coll. «Idées et recherches".

BARTHES, R. [1980]: La Chambre claire. Note sur la photographie, Paris, Gallimard/Seuil, coll. "Cahiers du cinéma».

BAzIN, A. [(1947) 1998] : "Paris 1900. À la recherche du temps perdu", L'Écran français, 30 septembre 1947, repris dans Le Cinéma français de la Libération à la Nouvelle Vague (1945-1958), textes réunis par J. Narboni, Paris, Petite bibliothèque des Cahiers du cinéma, 242-244.

Bertozzi, M. [2001]: "The Gaze and the Ruins: Notes for a Roman Film Itinerary", dans E. Bruscolini (dir.), Rome in Cinema: Between Reality and Fiction, Rome, Fondazione Scuola Nazionale di Cinema, Cineteca Nazionale, 14-27.

BRENEZ, N. [1998]: De la figure en général et du corps en particulier: l'invention figurative au cinéma, Paris, De Boeck Université.

CADAVA, E. [2001] : "Lapsus imaginis. The image in ruins", October, $\mathrm{n}^{\circ} 96$, printemps, 35-60.

CARAIOn, M. [2003]: Pour fixer la trace. Photographie, littérature et voyage au milieu du XIXe siècle, Genève, Droz.

CHerchi UsAi, P. [2001]: The Death of Cinema, History, Cultural Memory and the Digital Dark Age, Londres, British Film Institute.

DE KUYPER, E. [1992]: «Fragments de l'histoire du cinéma. Quelques remarques sur la problématique du fragment", Hors cadre, ${ }^{\circ} 10,42-48$. Deleuze, G. [(1977) 1996]: «L'actuel et le virtuel», Dialogues, Paris, Flammarion, coll. "Champs», 179-185;

—_ [1985]: Cinéma 2. L'Image-temps, Paris, Minuit;

[1990]: Pourparlers, Paris, Minuit.

Didi-Huberman, G. [2001]: «Montage des ruines ", Simulacres, n 5 , septembre-décembre, 8-17.

EPSTEIN J. [1929]: La Chute de la maison Usher (adaptation d'une nouvelle d'E. Poe), France, 63 min., noir et blanc.

HABIB, A. [2004]: "L'Amour, en ruines: notes sur quelques photogrammes du film de Bill Morrison Light is Calling", Intermédialités, n 4, "Aimer", automne, 157-162.

MAKARIUS, M. [2004], Ruines, Paris, Flammarion.

MÉnIEL, A. [1991]: L'Écran du temps, Lyon, Presses universitaires de Lyon, coll. "Regards et écoutes".

MORRISON, B. [2003] : Light is Calling, États-Unis, 8 min., noir et blanc, sans parole.

PAÏNI, D. [2002]: Le Temps exposé. Le cinéma, de la salle au musée, Paris, Cahiers du cinéma, coll. "Essais".

SCHEFER, J.-L. [1997]: Du monde et du mouvement des images, Paris, Cahiers du cinéma, coll. "Essais".

Smither, R. (dir.) [2002], This Film is Dangerous. A Celebration of Nitrate

Film, Bruxelles, Fédération internationale des archives du film.

STAROBINSKI, J. [1964]: L'Invention de la liberté (1700-1789), Genève, Albert Skira.

TOFFETTI, S. (dir.) [1992]: Yervant Gianikian, Angela Ricci Lucchi, Florence et Turin, Hopefulmonster editore et Museo nazionale del cinema, coll. "Cinemazero».

TOUlET, E. [1988]: Cinématographe, invention du siècle, Paris, Gallimard/ Réunion des musées nationaux, coll. "Découvertes". 